Cahiers de civilisation médiévale

\title{
Collectif, Medieval Clothing and Textiles
}

Sophie Jolivet

\section{OpenEdition}

\section{Journals}

Édition électronique

URL : https://journals.openedition.org/ccm/8064

DOI : $10.4000 / \mathrm{ccm} .8064$

ISSN : 2119-1026

\section{Éditeur}

Centre d'études supérieures de civilisation médiévale/Université de Poitiers

\section{Édition imprimée}

Date de publication : 1 septembre 2021

Pagination : 273-275

ISBN : 978-2-490783-10-6

ISSN : 0007-9731

Référence électronique

Sophie Jolivet, «Collectif, Medieval Clothing and Textiles », Cahiers de civilisation médiévale [En ligne], 255 | 2021, mis en ligne le 01 septembre 2021, consulté le 01 février 2022. URL : http:// journals.openedition.org/ccm/8064; DOI : https://doi.org/10.4000/ccm.8064

La revue Cahiers de civilisation médiévale est mise à disposition selon les termes de la Licence Creative Commons Attribution - Pas d'Utilisation Commerciale - Pas de Modification 4.0 International. 
Medieval Clothing and Textiles, Monica L. Wright, Robin Netherton et Cale R. Owen-Crocker (éd.), 15, 2019.

Le volume 15 de Medieval Clothing and Textiles, édité par Monica L. Wright, Robin Netherton, Cale R. Owen-crocker, est le dernier volume de l'excellente revue anglophone, réunissant sept importants articles traitant d'aspects variés et interdisciplinaires sur les vêtements et textiles, de la littérature à l'archéologie, en passant par l'histoire et l'histoire de l'art du Moyen Âge occidental. L'ouvrage comprend des illustrations nécessaires à la compréhension des contenus, essentiellement en noir et blanc. Le volume se termine par une série de comptes rendus d'articles et ouvrages internationaux intéressant la thématique, suivi des sommaires des précédents volumes de la revue.

L'ouvrage s'ouvre sur un intéressant panorama historiographique de la recherche dans le domaine de l'histoire des vêtements et des textiles, livré par C. Owen-Crocker (« Old Rags, New Responses: Medieval Dress and Textiles »/Vieux chiffons, nouvelles pistes : vêtements et textiles médiévaux, p. 1-31). Professeur émérite de l'université de Manchester, elle est l'une des fondatrices et éditrice de la revue Medieval Clothing and Textiles. Elle balaie les étapes importantes de la recherche qui ont jalonné sa carrière de chercheuse, depuis le milieu du $\mathrm{XX}^{\mathrm{e}} \mathrm{s}$., en pointant les perspectives futures. À grands traits, les sources de l'histoire du vêtement médiéval sont évoquées : vêtements précieux des trésors d'église, attributs royaux, tapisseries et broderies, artefacts archéologiques issus de tombes habillées ou de milieux humides, fragments de reliquaires, littérature, sculptures, peintures... Depuis les années 1990 et le développement des bases de données en ligne, les chercheurs ont acquis un accès plus aisé aux sources, ce qui a ouvert des facilités d'analyse, mais a également permis le développement de l'interdisciplinarité. Depuis 20 ans, un travail important a été réalisé sur le vocabulaire textile à l'échelle internationale : précisé et replacé dans son contexte, il a permis notamment d'identifier l'évolution des vêtements et de leurs usages dans le temps, mais aussi de revenir sur des termes non utilisés au Moyen Âge. Dans la deuxième moitié $\mathrm{du} \mathrm{Xx}^{\mathrm{e}} \mathrm{s}$., l'approche sociologique du vêtement a permis de mieux comprendre le vêtement médiéval dans son contexte. Les recherches d'aujourd'hui cherchent à préciser la singularité

des vêtements dans les différentes sources, liées à l'intention du porteur et/ou de l'auteur. La notion de « biographie de l'objet » replace chaque objet dans une micro-histoire individuelle, ouvrant sur des usages et des changements d'état qui dépassent le simple moment de sa création. Des technologies de pointe (analyses génétiques ou chimiques, explorations visuelles de tombes sans destruction...) ouvrent des perspectives riches, dans la mesure où les chercheurs pourront mener des travaux collectifs et interdisciplinaires.

Le deuxième article, proposé par Maren Clegg Hyer, « Text/Textile: « Wordweaving in the Literatures of Anglo-Saxon England ( Texte/textile : le « tissage des mots » dans la littérature anglo-saxonne, p. 33-51), propose de faire le lien entre le texte et le textile, deux mots qui ont la même origine, et le « tissage des mots » à travers la littérature anglo-saxonne. On sait que la métaphore du tissage pour désigner l'acte d'écriture est utilisée dès l'époque Augustinienne en latin, mais qu'en est-il en vieil Anglais? Cet article propose de rechercher les signes, dans la littérature anglo-saxonne, d'une « invention » du « tissage des mots » indépendante de celle de l'Italie augustinienne. Pour cela, l'a. inventorie les signes d'une proximité entre l'activité de tissage et celle de l'écriture, tant dans la vie quotidienne que dans la littérature. Ainsi la production textile étant omniprésente dans le paysage et dans la vie quotidienne de l'Angleterre, il n'est pas étonnant de trouver des similitudes évidentes entre le geste de l'écriture et le tissage et ses motifs. Dans la Vie de Dunstan, saint Anglo-saxon du VII ${ }^{\mathrm{e}}$ s., le saint est à la fois un poète et un dessinateur de motifs à broder. Les motifs à entrelacs ornent aussi bien les manuscrits que d'autres productions artisanales comme les métaux, l'ivoire, l'os, ainsi que les textiles. Cynewulf, l'un des rares poètes anglo-saxons dont les poèmes nous soient parvenus, explique son processus d'écriture en utilisant la métaphore textile. Au-delà, la manière même de composer et d'ordonner les mots, avec des césures, des allitérations ou des formules poétiques répétées et précisément placées tout au long du poème crée une « texture narrative" que les auditeurs pouvaient voir et entendre. Ainsi selon l'a., le « tissage des mots » tel qu'il est pratiqué par les anglo-saxons ne relève pas d'un héritage du 
latin, mais serait davantage une création en lien avec la culture locale.

Le troisième article reste dans le domaine de la littérature. Elisabeth M. Swedo, « Unfolding Identities: The Intertextuel Roles of Clothing in the Nibelungenlied and Völsunga Saga » (Le croisement des identités : les rôles intertextuels de l'habillement dans la chanson des Niebelungen et la Völsunga Saga, p. 53-77) analyse les convergences et divergences des usages symboliques du vêtement dans deux récits légendaires du XIII ${ }^{\mathrm{e}} \mathrm{s}$. : la chanson des Niebelungen, d'origine germanique d'une part et la Völsunga saga, d'origine islandaise d'autre part. Le vêtement sert plusieurs desseins dans la littérature médiévale : il situe visuellement le personnage, lui donne une identité; il reflète la personnalité intérieure; il signale l'évolution, le changement de statut; il dévoile les tensions dans les actes de tromperie. La société médiévale attache une grande importance à la convergence entre l'apparence et la réalité. L'a. relève certaines convergences qui situent les deux récits dans la même tradition culturelle : si dans la littérature française une personne peut tromper simplement en changeant de vêtements, ce n'est pas le cas ni dans les Niebelungen, ni dans la Völsunga saga : un personnage ne peut pas masquer ses qualités internes avec un vêtement inapproprié : ainsi lorsque Hjördis (sœur de la veuve de Sigurd) échange ses vêtements avec sa servante, son port noble et ses manières la trahissent. Dans les deux récits, il faut l'intervention de la magie pour que la dissimulation de l'identité soit effective. En cela les deux récits appartiennent à la même tradition culturelle. Parce que le vêtement reflète l'identité intérieure, changer de vêtements modifie non seulement le statut, mais aussi la personnalité du porteur. Pour les hommes, le changement est de l'ordre du rite d'initiation ou rite de passage de l'homme au guerrier. Pour les femmes, le processus de transformation renvoie à la question du genre : la « guerrière » (fonction masculine par excellence), femme vengeresse dotée d'une force prodigieuse, devient épouse (fonction féminine) soumise à l'autorité de l'homme, en enlevant son casque et en fendant son armure (Niebelungen). En cela le récit devient conforme en la croyance en la domination masculine qui a sans doute régi les opinions des auteurs et au moins la moitié de leur public. Une divergence entre les deux récits concerne le port de peaux de bêtes. Dans le récit de la Völsunga Saga, les deux frères
Sinfjötli et Sigmund revêtent des peaux de loup. Au-delà d'acquérir la force de l'animal, ce choix résonne avec les références culturelles des lecteurs islandais, qui font du dieu guerrier Odin, le « porteur de peau de loup ». De même dans les deux récits, les vêtements de chasse en peau de bêtes témoignent d'un rapport culturel différent à la Nature : lorsque Sinfjötli vêtu d'une peau de loup fait apparaître sa bestialité, sa proximité avec la bête, Siegfried revêt des peaux de bêtes qu'il a vaincues, en dominateur de la Nature.

Avec l'article suivant, on quitte la fiction pour étudier le vêtement à la cour du roi Jean d'Angleterre. Hugh M. Thomas, «Clothing and Textiles at the Court ok King John of England, 1199-1216 » (Les vêtements et les textiles à la cour du Roi Jean d'Angleterre, 1199-1216, p. 79-100) complète les connaissances techniques sur les vêtements de cour à partir de rouleaux de comptes découverts récemment, enregistrant les dépenses de la garde-robe du roi. Ces documents sont partiels et incomplets, mais apportent un nouveau regard sur l'habillement et les textiles d'une cour européenne. La gamme des achats textiles est étendue : vêtements, pavillons, couvertures de chevaux, textiles d'ameublement, nappes, draps de lits. Les fournitures de vêtements comprennent des tissus et des fourrures. Ainsi les données permettent de différencier les tissus de laine en fonction de leur prix. L'écarlate est la plus chère, certaines valant 20 jours de travail d'un laboureur. La soie, symbole de luxe, semble peu présente à la cour de Jean. Elle n'est pourtant pas absente à cette période car les églises en sont fréquemment ornées. L'a. interprète cette particularité comme un choix volontaire plutôt qu'une nécessité économique. De même, les fourrures les plus chères (zibelines et hermines) sont rares, au profit des écureuils (dont vairs), lapin et mouton. Les sources évoquent de manière très partielle les provenances, de même que les éléments concernant la taille ou le style des vêtements. Selon les standards de l'époque, la « robe » (en tant qu'ensemble de vêtements) standard comprend trois pièces, portés sur des sous-vêtements de lin : une tunique (tunica), un surcot (supertunica) et un manteau (capa, parfois pallium). Les vêtements sont réalisés sur mesure au sein de la cour. En dehors des données sur le roi lui-même, quelques données évoquent l'entourage : serviteurs, seigneurs, famille, dans le respect des hiérarchies sociales. L'a. conclut en soulignant que ces données 
permettent de mettre en lumière les goûts personnels du roi Jean en matière esthétique, notamment son appétence pour la variété et les couleurs. Tout comme les autres princes de son époque, Jean connaissait la valeur et le rôle du vêtement pour paraître riche et puissant.

Dans l'article suivant, Tina Anderlini, « Dressing the Sacred: Medallion Silk and Their Use in Western Medieval Europe » (Habiller le sacré : les médaillons de soie et leur utilisation en Europe occidentale médiévale, p. 101-136) s'intéresse aux usages de la soie aux motifs de médaillons en Europe médiévale, aussi bien dans l'archéologie, les textes, que dans l'iconographie. Dès l'époque byzantine, les motifs à médaillons sont présents, notamment dans le contexte des pratiques nuptiales. Représentés dans les manuscrits précieux, sur des murs d'églises ou de monastères, ils semblent jouer un rôle protecteur, mais aussi peut-être de représentation de «l'idée du Sacré », appliqué notamment à la famille royale. Les médaillons qui se multiplient au $\mathrm{XII}^{\mathrm{e}} \mathrm{s}$. sont en partie transmis via les routes commerciales ouvertes avec les royaumes chrétiens d'Orient, en provenance des mondes orientaux et musulmans. Pour l'a., le succès de ces médaillons s'explique par leurs connexions avec les images du pouvoir sacré que les princes chrétiens n'hésitent pas reprendre à leur compte, à l'instar du roi Roger II de Sicile dont le manteau d'apparat au motif de lions a été préservé. L'intérêt pour les médaillons de soie commence à décliner lorsque la " Pax Mongolica » entraîne un afflux de productions chinoises en Europe. Il est résiduel au $\mathrm{XIV}^{\mathrm{e}}$ et $\mathrm{XV}^{\mathrm{e}} \mathrm{s}$.

Alexandra Concha Sahli, « Habit Envy: ExtraReligious Groups, Attire, and the Search for legitimation Outside the Institutionalised Religious Orders » (L'envie d'Habit : groupes extra-religieux, tenue vestimentaire et recherche de légitimation en dehors des ordres religieux institutionnalisés, p. 137-156) enquête sur les stratégies vestimentaires des groupes extra-religieux, dans leur quête de légitimation aux
$\mathrm{XIII}^{\mathrm{e}}$ et $\mathrm{XIV}^{\mathrm{e}} \mathrm{s}$. Prendre l'habit religieux est considéré comme un acte d'engagement, mais aussi de reconnaissance et d'identification de l'ordre auquel le religieux appartient. Aussi les autorités religieuses sont particulièrement attentives à limiter les éventuelles usurpations. La question se pose de manière cruciale lorsque des groupes de laïcs, comme les pénitents où les biguines, cherchent à témoigner, notamment par leur apparence, d'une sorte de renonciation au siècle et d'un mode de vie dévot, sans pour autant être reconnus comme des ordres religieux. Ainsi l'Église multiplie les mises en garde et met en place des règles vestimentaires parfois extrêmement précises, sous peine d'excommunication. Le fait que ces individus, mi-laïcs mi-religieux, vivent « dans le siècle » les expose davantage que les religieux isolés dans leur monastère. Au final, la reconnaissance de ces groupes par l'Église passe par la reconnaissance de leurs signes distinctifs communs, mais elle apparaît bien comme un moyen de mieux les contrôler.

Joanne W. Anderson « The Loom, the Lady, and Her Family Chapels: Weaving Identity in Late Medieval Art » (Le métier à tisser, la dame et sa chapelle familiale : identité vestimentaire dans l'art médiéval tardif, p. 157-181) clôt la série d'articles, en étudiant une représentation de l'annonciation dans l'église de Sainte Madeleine de Rentsh (sud Tyrol), dans laquelle la Vierge est représentée avec un métier à tisser sur lequel un tissu héraldique au motif de Lion est en train d'être tissé. Cette représentation inhabituelle est interprétée à la lumière du contexte local : insérée dans une iconographie antérieure, elle évoque le patronage des familles Brandis/Botch, à un moment bien particulier de leur histoire : l'union de Randold de Brandis avec Margaret Botch, héritière de la famille florentine des Rossi, famille de marchands ayant fait fortune dans le commerce des textiles.

Sophie Jolivet UMR 6298 - Artehis/Université de Bourgogne 